\title{
Temperature-frequency scaling in amorphous niobium-silicon near the metal-insulator transition
}

\author{
Hok-Ling Lee, John P. Carini, and David V. Baxter \\ Department of Physics, Indiana University, Bloomington, IN 47405 \\ George Grüner \\ Department of Physics, University of California, Los Angeles, CA 90024
}

(June 24, 2021)

\begin{abstract}
Millimeter-wave transmission measurements have been performed in amorphous niobium-silicon alloy samples where the DC conductivity follows the critical temperature dependence $\sigma_{d c} \propto T^{1 / 2}$. The real part of the conductivity is obtained at eight frequencies in the range $87-1040 \mathrm{GHz}$ for temperatures $2.6 \mathrm{~K}$ and above. In the quantum regime $\left(\hbar \omega>k_{B} T\right)$ the real part of the high-frequency conductivity has a power-law frequency dependence $\operatorname{Re} \sigma(\omega) \propto \omega^{1 / 2}$. For temperatures $16 \mathrm{~K}$ and below the data exhibits temperaturefrequency scaling predicted by theories of dynamics near quantum-critical points.
\end{abstract}

72.15.Rh,72.30.+q,72.60.+g

The interplay between dynamics and statistical mechanics in quantum phase transitions suggests that experiments probing the frequency dependence of such systems will be useful in understanding the physics of quantum-critical points, where quantum fluctuations have diverging characteristic length and time scales, $\xi$ and $\xi_{\tau}$ respectively [1] 3. For example, recent studies of the quantum-Hall effect [4,5] and two-dimensional superconductor-insulator transition [6] have exploited this approach. The disorder-induced metal insulator transition in bulk systems has been studied for decades as a quantum-critical point [7,8] (and see [9] for a recent review), yet few experiments have studied the frequencydependent conductivity in the quantum regime, $\hbar \omega>$ $k_{B} T, \hbar / \xi_{\tau}$ (exceptions being far-infrared conductivity measurements in Si:P near its metal-insulator transition [10,11) or in the crossover regime where $\hbar \omega \simeq k_{B} T$.

Even without a microscopic model for the transition, several general predictions can be made on the basis of scaling arguments for the critical behavior of the conductivity as a function of temperature and frequency, $\sigma_{c}(T, \omega)$. For $\hbar \omega \gg k_{B} T$, one directly observes the quantum fluctuation conductivity, $\sigma_{c} \propto \omega^{1 / z}$ (where $z$ is the dynamical exponent); for $k_{B} T \gg \hbar \omega$, one expects [3] the thermal energy scale to limit the duration of quantum fluctuations resulting in a DC conductivity varying as $\sigma_{c} \propto T^{1 / z}$. For intermediate temperatures and frequencies, a universal crossover function that depends only on the ratio $\frac{\hbar \omega}{k_{B} T}$ interpolates between the limiting behaviors.
We report measurements of the millimeter-wave transmission through an amorphous niobium-silicon alloy sample as a function of temperature. This sample has a temperature dependence for its DC electrical conductivity indicating that the niobium concentration is very close to the critical value that divides alloys having zero DC electrical conductivity for $T \rightarrow 0$ (insulators) from those which conduct for $T \rightarrow 0$. The experiment spans a sufficiently broad range of frequency that it probes the quantum regime for the lowest experimental temperatures. The transmission data are analyzed to obtain the magnitude of the real part of the conductivity as a function of temperature and frequency. In the quantum regime, the data is consistent with a power-law frequency dependence for the conductivity $\operatorname{Re} \sigma \propto \omega^{1 / 2}$. For a range of temperatures from $2.6 \mathrm{~K}$ to $16 \mathrm{~K}$ we find that the temperature and frequency dependances of $\operatorname{Re} \sigma$ are consistent with the existence of a crossover function.

Amorphous niobium-silicon samples are grown onto 0.5 $\mathrm{mm}$ thick sapphire substrates using a sequential sputtering technique. The substrates are mounted on a rotating stage and are exposed alternately to pure silicon and niobium sources, which deposit bilayers of approximately 1 $\mathrm{nm}$ amorphous silicon and a sub-monolayer of niobium. This technique produces large (15 mm diameter), homogenous samples with thickness up to $0.5 \mu \mathrm{m}$, which are needed for the optical measurements. The rotational rate of the substrate over each source determines the niobium concentration of each sample. Low angle x-ray diffraction experiments demonstrate near-total interdiffusion of the niobium and silicon, consistent with previous reports of niobium-silicon multilayers [12]. In addition to this large sample, a sample suitable for dc electrical transport experiments is grown simultaneously in a Hall-bar configuration on an adjacent sapphire substrate.

The temperature dependence of the dc conductivity for our samples is very similar to that found near the critical niobium concentration in other studies of amorphous Nb-Si 13 16], which all found a temperature dependence for the DC critical conductivity $T^{1 / 2}$, implying $z=2$. For the sample used in the transmission measurement, the temperature dependence of the DC conductivity in the temperature range $1.4-15 \mathrm{~K}$ is $\sigma=\sigma_{0}+475 T^{1 / 2}\left(\Omega \mathrm{mK}^{1 / 2}\right)^{-1}$, where $\sigma_{0}=125(\Omega m)^{-1}$ (the absolute uncertainty is $5 \%$ ). The positive, small 
value for $\sigma_{0}$ implies that this sample is barely metallic and has a characteristic quantum energy scale that is small enough so that experiments for temperatures above $1 \mathrm{~K}$ (or frequencies with $\frac{\hbar \omega}{k_{B}} \gg 1 \mathrm{~K}$ ) are probing the quantum-critical behavior of this alloy system [3]. Tunneling experiments support the existence of a small characteristic quantum energy scale in similar samples 13,14. The conductivity for this sample begins rising above the $T^{1 / 2}$ dependence for $T>15 \mathrm{~K}$.

The millimeter-wave conductivity measurements are performed by placing a sample as one window of a movable holder, which also contains an empty window, in the tail of an optical cryostat. Several different backwardwave oscillator sources produce a few milliwatts of tunable monochromatic radiation in the frequency range 75-1080 GHz. During the experiment, the frequency is dithered by a few percent in order to average over interference oscillations in the transmission produced by multiple reflections between other objects in the optical path. Because the sapphire substrates are birefringent, a wiregrid in front of the sample polarizes the incident radiation along the sapphire $c$-axis (the higher-index axis). The transmission (the ratio of the power transmitted through the sample to that transmitted through the empty window) is measured as a function of frequency and temperature for both the sample and a blank substrate (for the same relative polarization as for the sample).

The transmission oscillates as a function of frequency because the substrate acts like a Fabry-Perot resonator with a rather low Q-factor (see the inset of Figure 1, the transmission of the sample at two temperatures as a function of frequency and for a substrate without a sample at room temperature). In principle, if the thickness $d_{1}$ and refractive index $n_{1}$ of the substrate and the thickness of the sample $d$ are known, then the complex conductivity of the film $\sigma(\omega)$ can be determined at a discrete set of frequencies from the magnitude of the transmission at each peak frequency $\left|t\left(\omega_{\text {peak }}\right)\right|^{2}$ and the shifts of the peak frequency value. In practice, experimental uncertainties make this inversion problematic; however in the limit that $\left(|\sigma| d Z_{0}\right) / n_{1} \ll 1$ (where $Z_{0} \approx 377 \Omega$ is the impedance for electromagnetic waves in vacuum) one finds this approximate linear relationship (accurate to 3\% or better in our experiment) from standard transmissionline theory:

$$
\operatorname{Re} \sigma\left(\omega_{\text {peak }}\right) \approx \frac{2}{Z_{0} d}\left(\frac{1}{\left|t\left(\omega_{\text {peak }}\right)\right|}-1\right) .
$$

This relation assumes no absorption in the substrate; as shown by the data for the substrate in the inset, absorption by the substrate alone becomes noticeable for the higher measurement frequencies, but correcting for its presence is straightforward.

The main panel in Figure 1 shows the peak transmission (corrected for absorption by the substrate) as a function of temperature for eight peak frequencies ranging from 87-1040 GHz. At low temperature, the transmission is much closer to unity for the lower frequencies than the higher frequencies, which shows that $\operatorname{Re} \sigma$ increases strongly with frequency in this frequency range. At higher temperature, the transmission is lower for all frequencies than at lower temperature (indicating that $\operatorname{Re} \sigma$ is greater for high temperatures) and the transmission values are approximately the same for the different frequencies, indicating that the conductivity is essentially independent of frequency at this temperature.

Figure 2 shows the frequency-dependence of the real part of the conductivity, plotted as a function of the square-root of frequency, for several temperatures in the range $2.8-32 \mathrm{~K}$. For the lowest temperatures $(2.8 \mathrm{~K}$ and $4.7 \mathrm{~K}$, where $k_{B} T<\hbar \omega$ except for the lowest frequency), the data follow a pure $\omega^{1 / 2}$ frequency dependence (as indicated by the straight line in the figure). For the intermediate temperatures (where $k_{B} T$ falls in the middle of the range of $\hbar \omega$ ), the data approach a similar $\omega^{1 / 2}$ dependence only for the highest frequencies and approach the dc values for lower frequencies. For $40 \mathrm{~K}$ (where $\left.k_{B} T>\hbar \omega\right)$ the increase in the conductivity with increasing frequency has vanished.

The systematic crossover from a frequency-determined to a temperature-determined conductivity when $k_{B} T \simeq$ $\hbar \omega$ suggests the existence of a crossover function $\Sigma$ of the form expected for quantum-critical dynamics [1.2]:

$$
\sigma\left(x_{c}, T, \omega\right)=C T^{1 / z} \Sigma\left(\frac{\hbar \omega}{k_{B} T}\right) .
$$

One can test for the existence of such a function using the dynamical exponent value $z=2$ and $C=$ $475\left(\Omega \mathrm{m} K^{1 / 2}\right)^{-1}$ (as suggested by the temperature dependence of the dc conductivity data at the critical point) and plotting the scaled conductivity data $\operatorname{Re} \sigma(T, \omega) /\left(C T^{1 / 2}\right)$ versus scaled frequency $\frac{\hbar \omega}{k_{B} T}$; the results are shown in Figure 3. The scaling procedure collapses the data over the entire frequency range for temperatures $16 \mathrm{~K}$ and below, indicating the existence of a single crossover function that depends only on the scaled frequency. Higher temperature data begins to rise above the collapsed data especially for lower frequencies. Varying the value of $z$ by more than $10 \%$ makes the collapse much less satisfactory.

The curve defined by the collapsed data has several features that a quantum-critical crossover function should possess. The demarcation between the flat part of the curve ( $T$-dominated dynamics) and the increasing part of the curve $\left(\omega\right.$-dominated dynamics) occurs for $\frac{\hbar \omega}{k_{B} T} \simeq 1$. This indicates that the thermal energy defines the timescale $\frac{\hbar}{k_{B} T}$ that limits the size of the quantum fluctuations (as measured by the frequency dependent conductivity), which is a key prediction of quantum-critical scaling theory. In addition, for high scaled frequencies, the crossover 
function follows a power-law dependence on scaled frequency with the same value for the dynamical exponent as was used to scale the vertical axis in the Figure. This means that one could have found a crossover function by scaling the conductivity data by $\omega^{1 / 2}$ and plotting the result as a function of $\frac{k_{B} T}{\hbar \omega}$.

An attempt to guess the form of the universal crossover function, $\Sigma=\operatorname{Re}\left(1-\imath b \hbar \omega /\left(k_{B} T\right)\right)^{1 / 2}$ where $b$ is supposed to be a dimensionless constant of order unity, is shown by the dashed line in Figure 3. This function describes the high and low frequency behavior of the collapsed data but misses the sharp crossover in the data for $\hbar \omega /\left(k_{B} T\right) \simeq 1$. This expression implies that there should be a sizeable imaginary component for the conductivity, which is negative in sign and, for exponent $1 / 2$, equal in size to the real part for high frequencies. The imaginary part of the conductivity can be extracted in this type of experiment from the shifts of the peak transmission frequencies; however these shifts turn out to be small and difficult to measure accurately when the index of refraction of the substrate is large (as it is here for sapphire, $3.35)$. For the four low frequency peaks $(85-340 \mathrm{GHz})$, we do observe peak shifts as a function of temperature that have the correct sign and the expected magnitude.

The fact that the scaling works only for temperatures $16 \mathrm{~K}$ and below is reasonable given the behavior of the $\mathrm{dc}$ conductivity for the sample, which begins to deviate from a $T^{1 / 2}$ temperature dependence for higher temperatures. This implies that there is an additional mechanism that becomes active for higher temperatures (electron-phonon interactions, for example) and a new time scale (in addition to $\frac{\hbar}{k_{B} T}$ ) that acts to limit the size of quantum fluctuations.

The value of the dynamic exponent $z=2$ is consistent with previous work on this alloy system close to the metal-insulator transition, including the temperature dependence of the dc conductivity [14,15] and tunneling measurements [13]. This value is different from what is expected from interacting models without a density of states singularity at the transition $(z=d$ the spatial dimension) or those with straight Coulomb interactions $(z=1)$. Models with a screened Coulomb interaction can produce $z=2$ [17,18]. Field-theoretical treatments also find several scenarios where the value $z=2$ is possible [9].

At least one other quantum-critical point has been investigated using combined frequency- and temperaturedependent conductivity experiments in the quantum regime, the magnetic field tuned transition between dif-

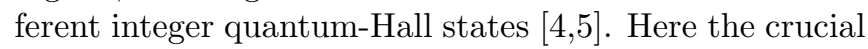
quantity was not the conductivity itself, since in a twodimensional system the critical conductivity approaches a constant of order $e^{2} / h$. Instead, the widths (as a function of magnetic field) of the peaks in the real part of the conductivity, which occur between the plateaux in the Hall resistance, were measured as a function of fre- quency and temperature. Analysis of the widths leads to the temperature-frequency scaling near the critical field magnetic value and a collapse of the data similar to what we observe. The dynamical exponent was found to be $z=1$. The crossover from temperature-dominated to frequency-dominated dynamics at the critical magnetic field appeared to occur for $\frac{\hbar \omega}{k_{B} T} \simeq \frac{1}{3}$ [5, which is somewhat different from the crossover location we observe.

In conclusion, our experiments on the temperature and frequency dependances of the conductivity of an amorphous niobium-silicon sample are consistent with the quantum-critical picture of the dynamics. In particular, from frequency-dependent experiments on a near-critical sample we find a crossover between a high-frequency regime dominated by quantum fluctuations and a lowfrequency regime dominated by thermal fluctuations. The crossover itself occurs for $\frac{\hbar \omega}{k_{B} T} \simeq 1$, which implies that the thermal energy directly limits the duration of the quantum fluctuations in the quantum-critical state. The form of the crossover function itself, in particular the location of the crossover and the dynamical exponent, have not yet been explained by theory.

Acknowledgements - We thank A. Schwartz and C. Hillman for assisting with the transmission experiments and D. Belitz, R. Bhatt, S. Girvin, T. Kirkpatrick, and S. Sachdev for fruitful conversations. JPC thanks the Aspen Center for Physics. This work was supported by NSF grants DMR-9314018, DMR-9423088, and DMR9503009 .

[1] S. Sachdev, to appear in Dynamical Properties of Unconventional Magnetic Systems, ed. A. Skjeltorp and D. Sherrington (Kluwer Academic, Dordrecht, 1997), condmat/9705266.

[2] K. Damle and S. Sachdev, Phys. Rev. B 56, 8714 (1997).

[3] S. L. Sondhi, S. M. Girvin, J. P. Carini, and D. Shahar, Rev. Mod. Phys. 69, 315 (1997) and references within.

[4] L. W. Engel, D. Shahar, C. Kurdak, and D. C. Tsui, Phys. Rev. Lett. 71, 2638 (1993).

[5] L. W. Engel, D. Shahar, C. Kurdak, and D. C. Tsui, in Proceedings of the 11th International Conderence on High Magnetic Fields in the Physics of Semiconductors, p 236, ed. D. Heiman (World Scientific, Singapore 1995).

[6] A. Yazdani and A. Kapitulnik, Phys. Rev. Lett. 74, 3037 (1995).

[7] P. W. Anderson, Phys. Rev. 109, 1492 (1958).

[8] F. J. Wegner, Z. Physik B 25, 327 (1976).

[9] D. Belitz and T.R. Kirkpatrick, Rev. Mod. Phys. 66, 261 (1994).

[10] T. F. Rosenbaum, K. Andres, G. A. Thomas, and P. A. Lee, Phys. Rev. Lett. 46568 (1981).

[11] A. Gaymann, H. P. Geserich, and H. v. Löhneysen, Phys. Rev. Lett. 71, 3681 (1993); Phys. Rev. B 52, 16486 
(1995).

[12] S. N. Song and J. B. Ketterson, Phys. Lett. A 155, 325 (1991).

[13] G. Hertel, D. J. Bishop, E. G. Spencer, J. M. Rowell, and R. C. Dynes, Phys. Rev. Lett. 50, 743 (1983).

[14] D. J. Bishop, E. G. Spencer, and R. C. Dynes, Solid-State Elec. 38, 73 (1985).

[15] L. C. Allen, M. A. Paalanen, and R. N. Bhatt, Europhys. Lett. 21, 927 (1993).

[16] J. P. Carini, H.-L. Lee, and D. V. Baxter, Ferroelectrics 176, 239 (1996).

[17] W.L. McMillan, Phys. Rev. B 24, 2739 (1981).

[18] A. L. Efros and B. I. Shklovskii, in Electron-Electron Interactions in disordered systems, ed. A. L. Efros and M. Pollak (Elsevier 1985), 409.

FIG. 1. Peak transmission (corrected for absorption in the substrate) is plotted as a function of temperature for eight peak frequencies ranging from 87 to $1040 \mathrm{GHz}$ for a $480 \mathrm{~nm}$ thick Nb-Si sample. The uncertainty for the transmission measurement is 0.02. Inset: Transmission as a function of frequency through a substrate with no sample (dotted line) and the sample for two temperatures: $2.8 \mathrm{~K}$ (thin solid line) and $40 \mathrm{~K}$ (thick solid line). The oscillations are caused by standing waves in the sapphire substrate.

FIG. 2. Re $\sigma(\omega)$ is plotted against the square root of the frequency for several temperatures in the temperature range 2.8 to $32 \mathrm{~K}$ and over the frequency range $87-1040 \mathrm{GHz}$. The uncertainty in the conductivity is $150(\Omega \mathrm{m})^{-1}$. For the lowest temperatures and when $\hbar \omega>k_{B} T$, the data follow a $\omega^{1 / 2}$ dependence (as indicated by the straight line). The data approach the corresponding dc values (plotted on the vertical axis) if $\hbar \omega \ll k_{B} T$. For the highest temperatures, the conductivity is approximately independent of frequency in this range.

FIG. 3. Log-log plot of scaled conductivity data versus scaled frequency with the factor $C=475\left(\Omega \mathrm{m} \mathrm{K}^{1 / 2}\right)^{-1}$. The uncertainty varies inversely with $T^{1 / 2}$, ranging from $20 \%$ at $2.6 \mathrm{~K}$ to $6 \%$ at $25 \mathrm{~K}$. When temperature is $16 \mathrm{~K}$ and below, the data for the entire frequency range collapse into one curve within the experimental noise. Higher temperature data begin to rise above the collapsed data systematically for low scaled frequencies. The trial scaling function (dashed line) was adjusted to match approximately the slope of the collapsed data for high frequencies but it does not describe the data well for $\frac{\hbar \omega}{k_{B} T} \simeq 1$. 


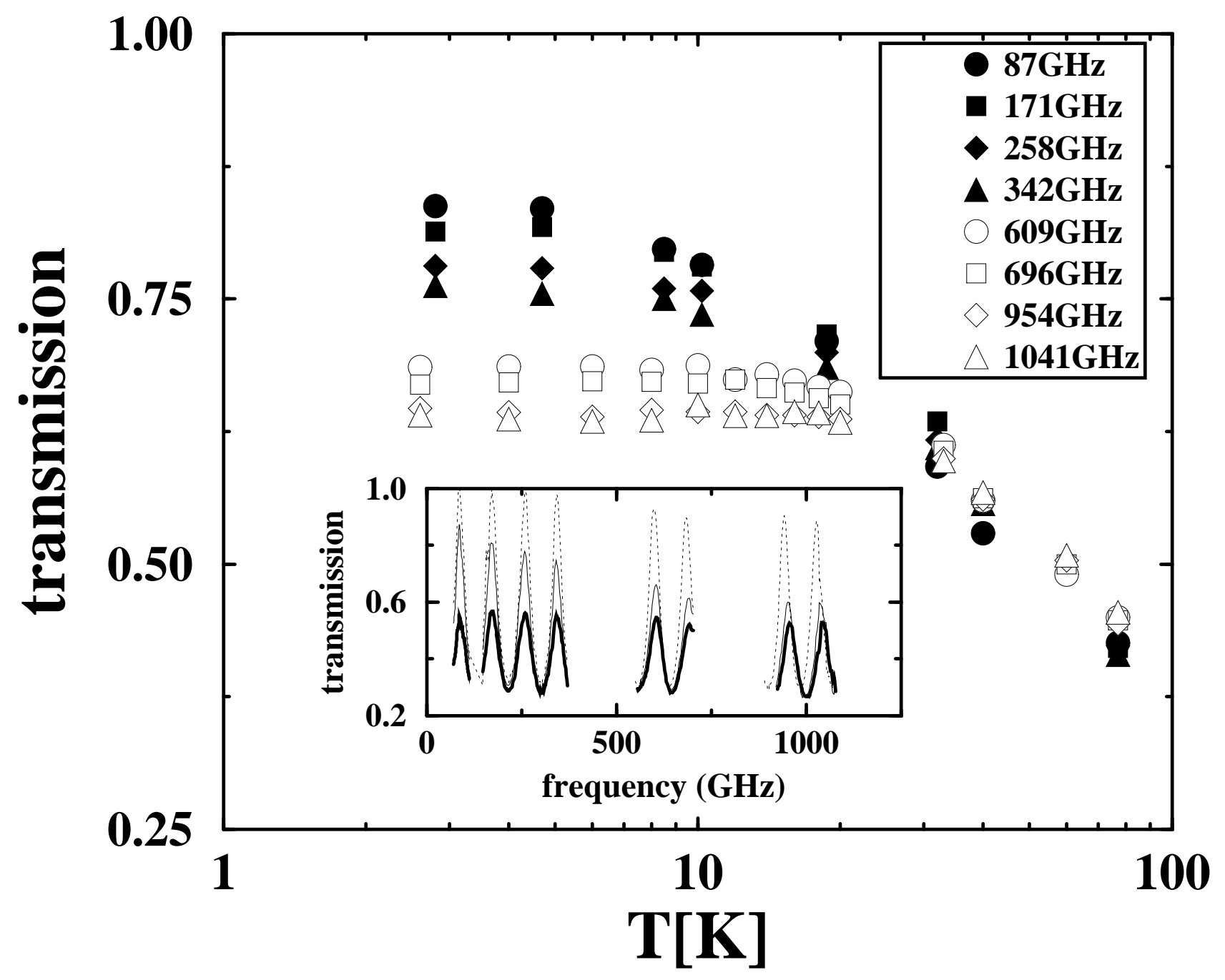

Figure 1

H.-L. Lee et al

Temperature-frequency scaling in $\mathrm{Nb}$-Si 


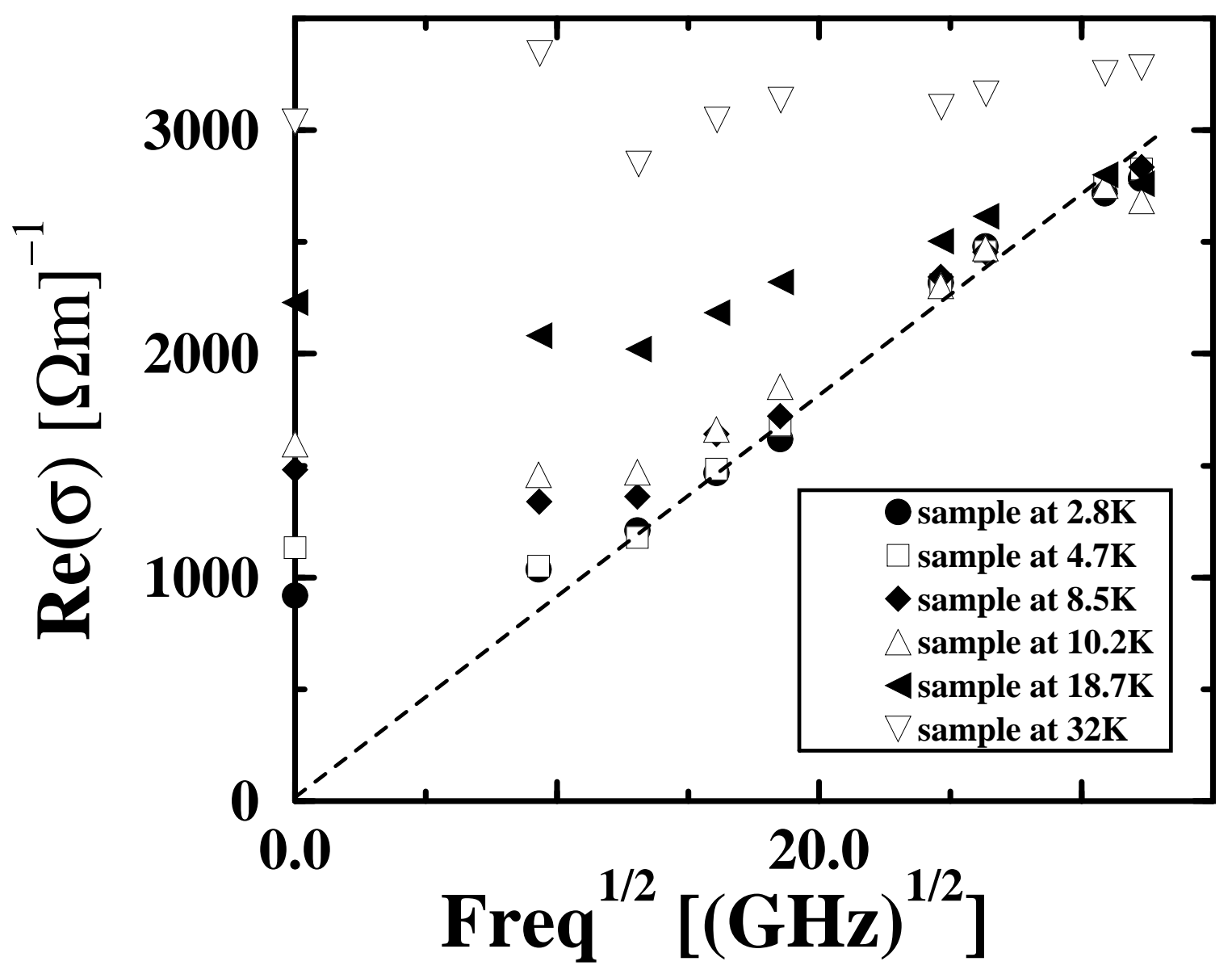

Figure 2

H.-L. Lee et al

Temperature-frequency scaling in $\mathrm{Nb}$-Si 


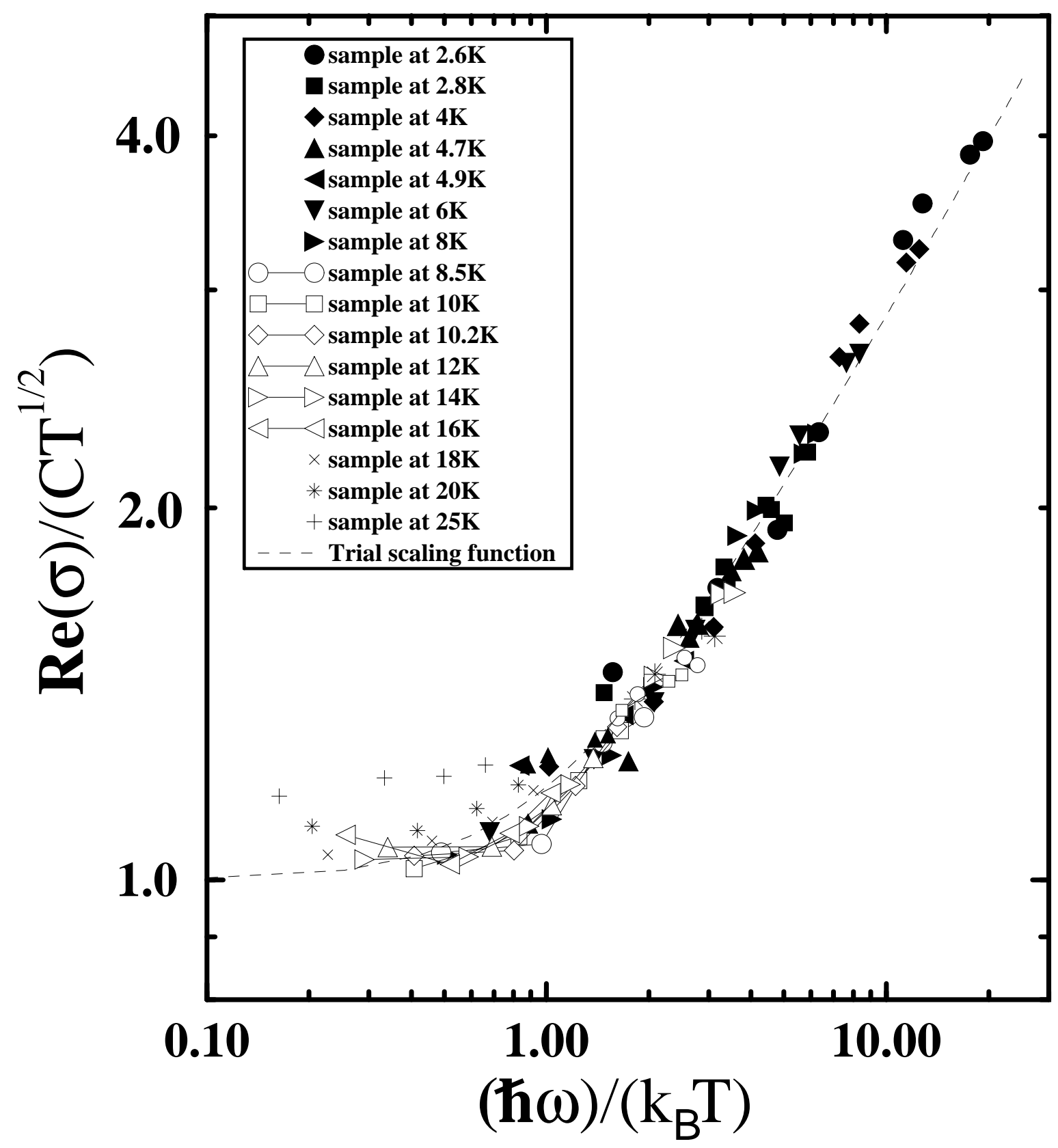

Figure 3

H.-L. Lee et al

Temperature-frequency scaling in $\mathrm{Nb}$-Si 